\title{
Selection of thummi specific chromosome regions in the progeny of Chironomus thummi thummix Chironomus thummi piger hybrids after long term culturing as a consequence of gonadal sterility
}

\author{
Klaus Hägele and \\ Helmut Speier
}

\begin{abstract}
Lehrstuhl für Genetik, Ruhr-Universität Bochum, Postfach 1021 48, 4630 Bochum 1, Federal Republic of Germany.
\end{abstract}

\begin{abstract}
Hybrids between two of our Chironomus th. thummi and $C h$. th. piger laboratory stocks are only fertile in the cross

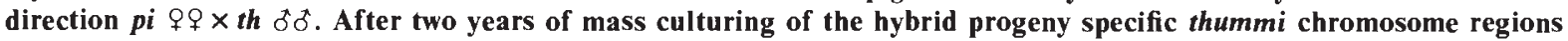
have accumulated up to the fivefold in the population, whereas the homologous piger regions tended to be eliminated. In a series of crosses we tested whether individuals occur in the mass culture which show the same kind of gonadal sterility as do the sterile $t h q \uparrow \times p i \precsim \measuredangle$ hybrids and whether this could be the reason for the unequal distribution of the chromosome regions at the end of the culturing period. In crosses where the mothers are thummi and the fathers are of hybrid origin 19 per cent of the female progeny are sterile on the basis of rudimentary developed gonads. Gonadal sterility of individuals is also observed when the mothers are hybrids. Here, between 10-13 per cent of the offspring are affected, irrespective of which males the hybrid females are mated with. It is concluded that in these cases the chromsomal and cytoplasmic egg constitution of the hybrid mother alone determines sterility, whereas in cases of maternal thummi constitution paternally derived piger but not thummi chromosomes induce sterility. The latter situation is suggested to be predominantly responsible for the unequal distribution of the thummi and piger chromosome regions observed after long term culturing of the hybrid progeny.
\end{abstract}

\section{INTRODUCTION}

Chironomus th. thummi and $C h$. th. piger are very closely related Chironomids which can be easily hybridised under laboratory conditions (Keyl, 1957). In natural populations of these sympatric forms hybrids are rarely found because of their reproductive isolation (Miehlbradt and Neumann, 1976). Both Chironomids are morphologically nearly undistinguishable, only their polytene chromosomes allow an exact taxonomic identification. The four polytene chromosomes of both Chironomids show the same banding pattern. However, within and near the centromeric regions between homologous thummi and piger chromosomes structural differences occur. These differences are due to an increased DNA content of specific thummi bands in comparison to the homologous ones in piger. Hereby, thummi bands contain $2^{n}$ times $(n=1,2,3,4)$ as much DNA as the homologous piger bands and, as a consequence, thummi has approximately 30 per cent more DNA than piger (Keyl, 1965). In both subspecies anAT rich DNA fraction ( $80^{\circ} \mathrm{C}$-th-DNA) has been identified (Schmidt et al., 1980). In situ hybridisation has shown that this DNA fraction is located in those bands of thummi which have a higher DNA content in comparison to the homologous piger ones. In piger, however, the $80^{\circ} \mathrm{C}$-th-DNA is present only in the centromeric bands (Schmidt et al., 1980). Up to now neither the function of the increased DNA fraction is known nor, in general, the genetic implication of the structurally different chromosome regions for both thummi and piger.

In salivary glands of the hybrids the structurally different chromosome regions are not somatically 
synapsed. Therefore, these regions can easily be identified as belonging to thummi or piger. After generations of sib mating of the hybrid progeny, the thummi and piger constitution of the structurally different chromosome regions is largely preserved, because between these regions crossing over frequencies are very low (Keyl, 1963). The aim of this study was to see whether the structurally different chromosome regions either of thummi or of piger origin are preferentially inherited in long term cultures of the hybrid progeny and to obtain information about the genetic implications of these regions for both subspecies.

\section{MATERIALS AND METHODS}

Chironomus thummi thummi (stock $\mathrm{Hl}$ ) and Chironomus thummi piger (stock $E$ ) were raised in the laboratory in mass cultures as already described (Hägele, 1975). For hybridisation of these two subspecies reciprocal crosses were made. The hybrids were then sib mated. Progeny could be obtained only if hybrids of the cross piger $q+q \times$ thummi of were used, the hybrids of the reciprocal cross showed sterility in both sexes (Hägele, 1984). For sib mating $100 \% q$ and $100 \delta \delta$ hybrid midges were transferred into a mass culture arrangement. Their progeny were continuously raised during two years without any change in the culturing conditions, and in the same way as the parental thummi and piger stocks. In mass cultures a generation cycle lasts about 30 days. Thus, culturing of two years corresponds to about 24 generations. Exact numbers of generations can, however, not be given because of the variation in growth of Chironomus larvae even from the same egg mass. This means that already after a short culturing period a mixed population of larvae of all four instar stages and imagines is produced. The generation numbers given in the text must therefore be seen as approximate values.

For eighteen consecutive times always at intervals of forty days about 200 fourth instar larvae were removed at random from the hybrid mass culture. The salivary gland chromosomes of these larvae were prepared and the chromosome complement of each larva was studied with respect to homozygosity or heterozygosity of the structurally different thummi and piger chromosome regions. With our standard stocks of Ch. th. thummi $(H 1)$ and $C h$. th. piger $(E)$ the following crosses were made, the adults were dissected and the developmental stage of their gonads was studied.



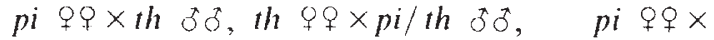

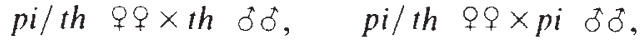

The abbrevations used here are th for $C h$. th. thummi and pi for Ch. th. piger.

\section{RESULTS}

The salivary gland chromosome complement of the hybrids is shown in fig. 1. After sib mating of the $C h$. th. piger $q 9 \times C h$. th. thummi of hybrids the theoretically expected distribution of the structurally different thummi and piger chromosome regions in the population of the progeny is 1
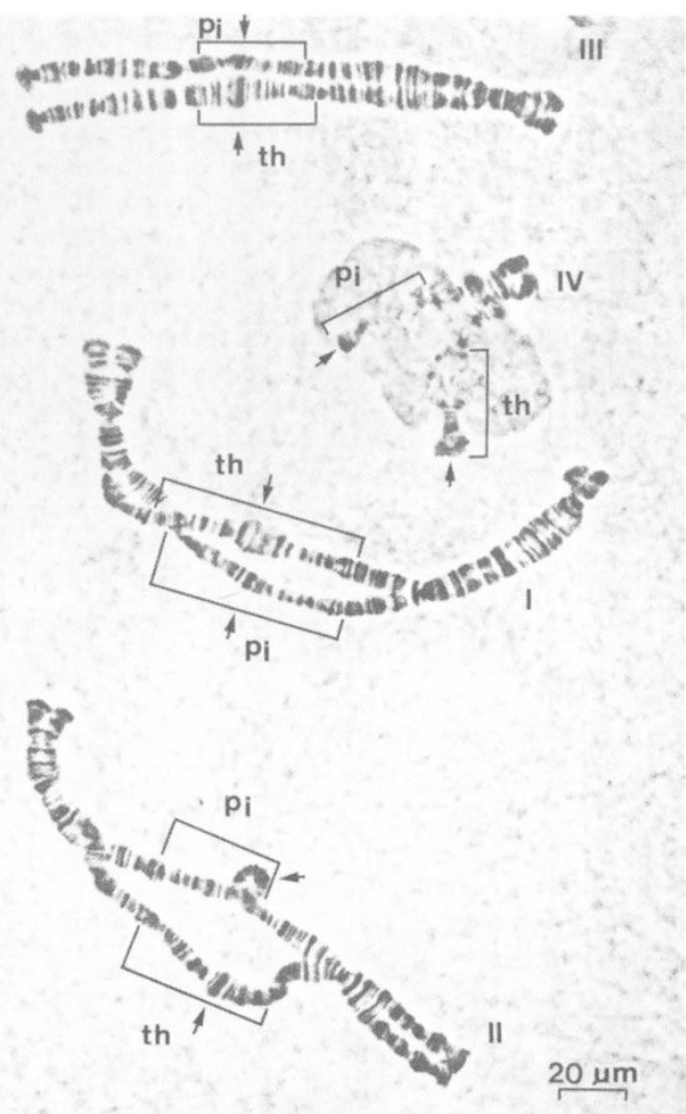

Figure 1 Salivary gland chromosome complement of a $C h . t h$. thummi $\times C h$. th. piger hybrid. Brackets indicate the structurally different chromosome regions of thummi and piger. th - thummi, $\mathrm{pi}=$ piger. Arrows point to centromere regions. 
$(t h: t h): 2(t h: p i): 1(p i: p i)$ for all four chromosomes. These theoretically expected distribution values are realised in the population for chromosome II and III until 23 weeks after the first sib mating $\left(U_{1}-U_{4}\right.$, fig. $\left.2 \mathrm{~b}, \mathrm{c}\right)$ and for chromosome IV until 45 weeks $\left(U_{1}-U_{8}\right.$, fig. 2d). These time

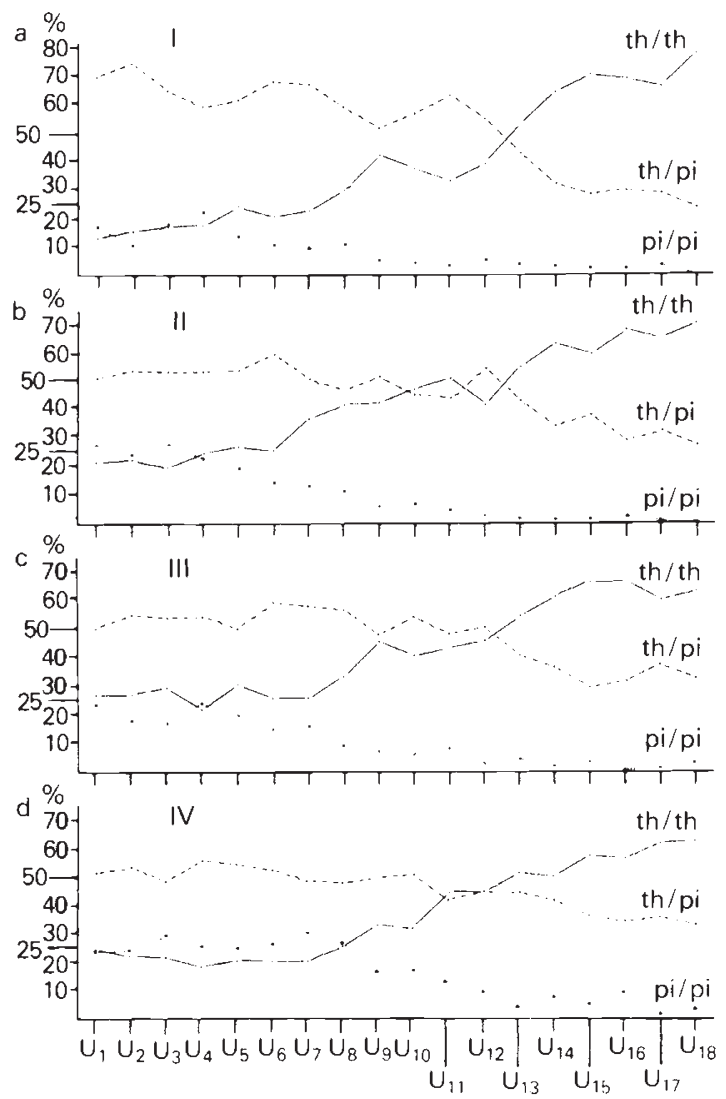

Figure 2 Distribution (in per cent) of homozygous thummi chromosome regions $(t h / t h \cdot-\cdot)$, homozygous piger regions $(p i / p i * \cdots *)$ and heterozygous thummi-piger regions $(t h / p i \circ \ldots \circ)$ in chromosomes I, II, III and IV during two years of mass culturing of the pi $q q \times t h$ to hybrid progeny. At each U 200 larvae were removed and the salivary gland chromosomes were studied. The time interval between two $U$ 's was always 40 days.

periods correspond to about 5 and 11 generation cycles respectively. The numbers of homozygous and heterozygous thummi and piger chromosomes I already show strong deviations from the expected $1: 2: 1$ distribution in the first generation. The number of individuals carrying heterozygous thummipiger chromosomes I is unusually high (70 per cent) and the number of those ones having homozygous chromosomes $\mathrm{I}$ is low (15 per cent in the average, $U_{1}$, fig. 2a). The theoretical $1: 2: 1$ distribution has never been realized for chromosome $I$ in the population during the two years of culturing (fig. 2a).

As shown in fig. 2, 28 weeks after the first sib mating of the hybrids ( $U_{5}$, sixth generation) the proportion of homozygous piger chromosomes I, II and III declines continuously in the mass culture reaching values lower than 3 per cent at the end of the second culturing year $\left(U_{18}\right.$, fig. $\left.2 \mathrm{a}, \mathrm{b}, \mathrm{c}\right)$. In contrast to this, homozygous thummi chromosomes accumulate steadily, after two years they reached 76 per cent in chromosome I, 70 per cent in chromosome II and 64 per cent in chromosome III $\left(U_{18}, 24\right.$ th generation, fig. $\left.2 \mathrm{a}, \mathrm{b}, \mathrm{c}\right)$. The decline of homozygous piger chromosome regions and a corresponding accumulation of homozygous thummi regions is also to be found in chromosome IV. However, this tendency begins 6 to 7 generations later than in the other chromosomes $\left(U_{8}\right.$, fig. 2d). At the end of the culturing period all thummi chromosome regions which are structurally different from their homologous piger regions have accumulated up to about fivefold that of the corresponding piger chromosome regions.

During culturing of the piger $q+\times$ thummiot $\delta$ progeny individuals should occur which originate from the same or nearly the' same parental (chromosomal and cytoplasmic) constitution as do the sterile $t h q q \times p i$ of hybrids. Characteristically, sterility of these hybrids is caused by a bilaterally drastic reduction in size of the gonads. The ovaries of the females for example, contain no eggs or only a few small ones (fig. 3). To test whether in the pi $q+9 \times$ th $\delta \delta^{*}$ hybrid progeny individuals show the same kind of gonadal sterility and whether this

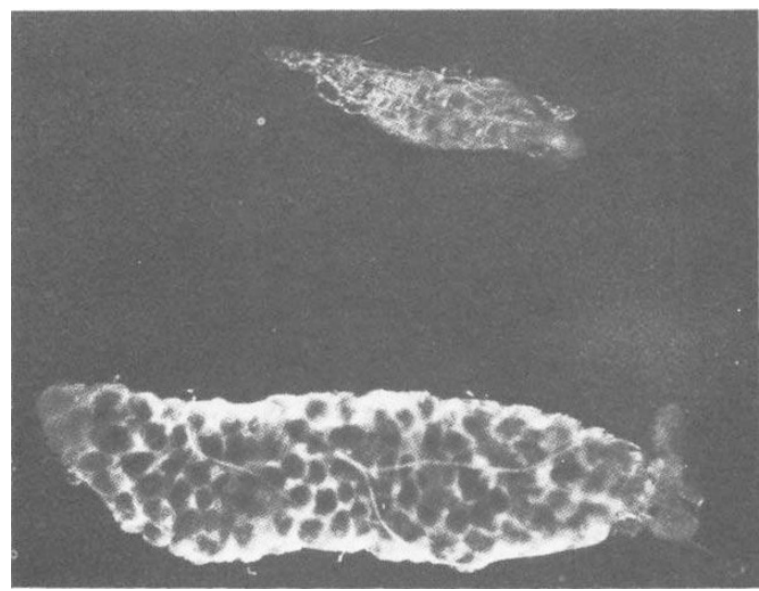

Figure 3 Ovaries of adult th $q q \times p i / t h$ of females. Top: Rudimentary developed gonad of a sterile female. Bottom: Normally developed gonad of a fertile female. 
Table 1 Distribution of females with bilaterally rudimentary developed ovaries among nine different crosses

\begin{tabular}{llll}
\hline Cross & & $\begin{array}{l}\text { No. of } \\
\text { females }\end{array}$ & $\begin{array}{l}\text { with } \\
\text { rudimentary } \\
\text { developed } \\
\text { ovaries }\end{array}$ \\
qq & \multicolumn{1}{c}{ of } & 108 & - \\
\hline$p i$ & $\times p i$ & 110 & - \\
$p i$ & $\times t h$ & 265 & - \\
$p i$ & $\times p i / t h$ & 340 & $10 \cdot 0$ \\
$p i / t h$ & $\times p i$ & 143 & $13 \cdot 3$ \\
$p i / t h$ & $\times t h$ & 254 & $13 \cdot 4$ \\
$p i / t h$ & $\times p i / t h$ & 176 & $100 \cdot 0$ \\
$t h$ & $\times p i$ & 609 & - \\
$t h$ & $\times p i / t h$ & 136 & \\
$t h$ & $\times t h$ & & \\
\hline
\end{tabular}

sterility could be the reason for the accumulation of the thummi chromosome regions described, ovaries of the female offspring of a series of crosses were studied. Three different categories of reproductive capacity can be distinguished in the crosses (table 1). (1). In cases where the mothers are piger and the fathers either piger, thummi or hybrids, 100 per cent of the female progeny has normal developed ovaries. (2). In cases of hybrid mothers nearly the same percentages (10-13 per cent) of the daughters are sterile due to rudimentary ovaries, irrespective whether the female parent mates with thummi or piger or hybrid males. (3). In cases of thummi females the percentage of daughters with rudimentary developed ovaries varies, depending to which males they mate.

The percentages of sterile males are very difficult to obtain in the Chironomids under study. Dissection of the testis does not always give a reliable indication of sterility, because males without sperms and ones with a reduced number were found. To test male sterility by further crossing is also not reliable because swarm formation of at least 20-30 $\delta^{*} \delta^{*}$ is a prerequisite for mating. During swarming of the males it is impossible to observe whether or not mating of a single male was successful, and both can result in a nondeveloping egg-mass.

\section{DISCUSSION}

In the progeny of Ch. th. piger $q 9 \times C h$. th. thummi $\delta \delta$ hybrids the structurally different thummi and piger chromosome regions of all four chromosomes were unequally transmitted during two years of culturing. During this time ( 24 generations) the original $1: 1$ relation of the thummi and piger chromosome regions in the hybrids changed for all chromosomes to a thummi-piger relation of 4.9:1 on average. Hereby, the accumulation of the thummi chromosome regions and the decline of the homologous piger regions in chromosome I, II and III becomes obvious five generations after the first sib mating of the hybrids and in chromosome IV after eleven generations. This process is continuous and can be easily observed because the identity of the structurally different thummi and piger chromosome regions is preserved in most individuals as a result of the low crossing over frequencies between the thummi and piger regions concerned $(0.7$ per cent for chromosome I, II, III, 7 per cent for chromosome IV, Keyl, 1963). The reason for the change of the $1: 1$ relation between thummi-piger chromosome regions could be gonadal sterility of specific individuals. As already mentioned, $p i$ $\$$ 우 $\times$ th $\delta$ o hybrids are totally fertile in both sexes, whereas the reciprocal cross is 100 per cent sterile (Hägele, 1984). This sterility is caused by rudimentary developed gonads. Hybrids of crosses in both directions have the same genomic constitution. Therefore, sterility of the $t h q q \times p i$ ot hybrids may be caused by interactions between paternal piger chromosomes or chromosome regions and the maternal thummi chromosome and cytoplasmic constitution, wherein piger chromosomes should be the inducers and the thummi constitution of the egg the reactive part for the production of sterility. This can be deduced from the fact that in those crosses where the females are of thummi constitution and the males possess differing numbers of piger chromosomes, the percentages of offspring with rudimentary developed gonads increases with the increasing number of piger chromosomes present in the paternal genome. Preliminary results, obtained from the progeny of matings of $t h q q$ with backcross $\delta \delta^{t}$ of th/pi hybrid chromosome constitution showed that individuals with normally developed gonads received from their fathers either none or only one piger chromosome, whereas their sterile sisters possessed four or three of them (Hägele, in preparation). Thus, piger chromosomes are more strongly eliminated from being transmitted than thummi chromosomes. Such cross situations as described above should occur after a few generations in the mass culture and they must be, along with similar crosses not studied, responsible for the decline of the piger chromosome numbers observed after long term culturing.

Hybrid females with a pi $+q \times t h$ of $\delta$ constitution always produce between $10-13$ per cent sterile offspring, irrespective whether they mate with piger or thummi or hybrid males. This result in the 
( $p i / t h) ~ q q \times t h$ to backcross is surprising, because interactions between paternally derived thummi chromosomes and the maternal hybrid constitution are not expected. This is because 100 per cent of the offspring of those crosses are fertile in which the individuals receive paternal thummi chromosomes and develop in eggs either of thummi or piger constitution. Thus, in cases of hybrid $p i q q \times$ th $\delta \hat{\delta}$ females it seems to be reasonable to assume that the chromosomal and cytoplasmic constitution of the eggs which they produce alone determines sterility of the offspring. Such a situation is described in the SF system of hybrid dysgenesis in Drosophila melanogaster (Kidwell, 1983). In Chironomus further investigations are under way. Sterility of some offspring of $p i$ 우 $\times t h$ o $\delta$ mothers should not influence the thummi or piger chromosome distribution during the culturing of the hybrid progeny, because the probability for thummi and piger chromosomes not to be transmitted should be the same.

The paternal derived factors for sterility induction could be located within those piger chromosome regions which are structurally different in comparison to thummi. As already mentioned between these regions of chromosome I, II and III, crossing over frequencies are 0.7 per cent on the average (Keyl, 1963). Therefore, these regions are transmitted unaltered in most individuals and can function as an unit during the whole culturing period. Alternatively, the chromosomal factors for sterility induction could be located in those piger regions which are structurally identical with thummi. However, between these thummi and piger regions crossing over frequencies are about 30 per cent. These frequencies should soon cause a highly mixed sequence of thummi and piger chromosome sections within each chromosome and would not explain the continuing elimination of the piger regions concerned, once linkage equilibrium is reached.

Up to now, it is not known whether all four piger chromosome regions work additively in inducing sterility or whether specific chromosome combinations must be present. There may be differences between individual chromosomes as suggested by the behavior of chromosome IV when compared with the other chromosomes. In chromosome IV the relation of the structurally different thummipiger regions appears to change 5-6 generations later than in chromosome I, II and III. Whether or not the crossing over frequency between the structurally different thummi and piger regions of chromosome IV, which is ten times higher than in the corresponding regions of the other three chromosomes, has any implication in this connection, is not yet clear.

\section{REFERENCES}

HÄGELE, K. 1975. Chironomus. In King, R. C. (ed.) Handbook of Genetics. Vol. 3, Plenum Press, New York, London, pp. 264-278

HÄGELE, K. 1984. Different hybrid effects in reciprocal crosses between Chironomus thummi thummi and Chironomus thummi piger including spontaneous chromosome aberrations and sterility. Genetica, 63, 105-111.

KEYL, H.-G. 1957. Untersuchungen am Karyotypus von Chironomus thummi. I. Karte der SpeicheldrüsenChromosomen von Chironomus th. thummi und die cytologische Differenzierung der Subspecies Ch. th. thummi und Ch. th. piger. Chromosoma, 8, 739-756.

KEYL, H.-G. 1963. Crossing over bei Bastarden von Chironomus thummi piger $\times$ Ch. thummi thummi. Chromosoma, 13, 588599.

KEYL, H.-G. 1965. Duplikationen von Untereinheiten der chromosomalen DNS während der Evolution von Chironomus thummi. Chromosoma, 17, 139-180.

KIDWELL, M. G. 1983. Intraspecific hybrid sterility. In Ashburner, M., Carson, H. L. and Thompson, J. N. Jr. (eds.) The Genetics and Biology of Drosophila. Vol. 3c, Academic Press, London, New York, pp. 125-154.

MIEHLBRADT, J. AND NEUMANN. D., 1976. Reproduktive Isolation durch optische Schwarmmarken bei den sympatrischen Chironomus thummi und Ch. piger. Behaviour, 58, 272-297.

SCHMIDT, E. R., VISTORIN, G. AND KEYL, H.-G., 1980. An AT rich DNA component in the genomes of Chironomus thummi thummi and Chironomus thummi piger. Chromosoma, 76, 35-45. 